\title{
Coping with the unthinkable: Psychosocial advances in the management of primary brain tumour
}

Tamara Ownsworth

School of Applied Psychology and Menzies Health Institute Queensland, Griffith University, Australia

Short heading: ASSBI Presidential Address 2016

Contact for correspondence:

A/Prof Tamara Ownsworth

School of Applied Psychology

Griffith University

Mt Gravatt, 4122

Australia

Email: t.ownsworth@griffith.edu.au

Ph: +61 737353307

Fax: +61 737353388 


\begin{abstract}
Brain cancer is a complex and distressing illness with a typically poor prospect for survival. Worldwide, approximately 256,000 people (1800 in Australia) are diagnosed each year (Ferlay, Soerjomataram \& Ervik, 2013). Cancer of the brain poses a double threat - to one’s survival and sense of self. Most people with primary brain tumour develop serious neurocognitive symptoms (e.g., seizures, memory loss) and experience poor mental health and quality of life, which places enormous burden on family caregivers. Not surprisingly, high rates of psychological distress have been reported by people with brain tumour and their family caregivers. Psychological distress can persist beyond primary treatment, and often increases in the long-term phase of illness due to the perceived threat and experience of recurrence and functional decline. Over the last decade there have been many key advances in the psychosocial management of people with brain tumour. This paper provides an overview of the functional, psychological and social consequences of brain tumour, summarises some leading developments in psychological assessment and management and outlines priorities for future intervention research.
\end{abstract}




\section{Introduction}

A primary brain tumour is a mass of atypical cells that originate in the brain or meninges (Mellado-Calvo \& Fleminger, 2009). Each year approximately 256,000 people $(3.4 / 100,000)$ worldwide are diagnosed with primary malignant brain and central nervous system cancers and approximately 189,000 die from such cancers (Ferlay, Soerjomataram \& Ervik, 2013). Worldwide rates of benign brain tumour are not recorded; however, based on US data, the incidence is estimated at 6.83 per 100,000 (Ostram et al., 2014). Although brain tumour is rare compared to other cancers (e.g., prostate: 148/100,000; breast: 113/100,000), five-year survival rates are much lower (22\%) compared to all cancers combined (66\%) (Australian Institute of Health \& Welfare, 2014). Similarly, for children aged under 16 years, brain cancer has the highest fatality and accounts for approximately one third of cancer related deaths within this age group. Brain tumours can develop at any age; however, most are diagnosed in middle adulthood (median age 55-59 years) (Ferlay et al., 2013). Accordingly, this paper focuses on psychosocial management for adults with primary brain tumour. For a review on the psychological impact of brain tumour in childhood refer to Fuemmeler, Elkin, and Mullins (2002).

There are an estimated 120 types of brain tumour; approximately 63\% are noncancerous or benign and 37\% are cancerous or malignant (Ostrom et al., 2014). Classification of brain tumours is based on the cells or tissue in which the tumour arises and the grade or degree of malignancy (Louis et al., 2007). The most common tumour type is benign meningioma (35\%) followed by gliomas (e.g., astrocytoma, oligodendroglioma), which represent $30 \%$ of all tumours. Gliomas develop in glial cells and are classified into grades according to histological appearance. Approximately 20\% of gliomas are low grade (Grades I-II) and $80 \%$ are high grade (Grade III-IV). Of the high grade or malignant tumours, approximately one quarter are anaplastic (Grade III) and the remainder are glioblastomas 
(Grade IV). These tumours rapidly infiltrate nearby brain tissue and are associated with the poorest prognosis, with a median survival time of 4 and 1.5 years respectively (Ostrom et al., 2014). Although people with low grade glioma typically have a better prognosis for survival (i.e., median of 7 years), there is a great deal of uncertainty associated with these tumours because these often recur or progress to malignant status (Lamborn et al., 2008). Overall, length of survival time depends on many interacting factors, including the type and location of the tumour, genetics, treatment regime and age (Van Meir, Hadjipanayis, Norden, Shu, Wen \& Olsen, 2010). Efforts are currently underway using genomic technology to improve the accuracy of classifying brain tumours (particularly glioblastomas) according to molecular subtypes in order to provide targeted therapeutics or personalised treatment (see Aldape, Zadeh, Mansori, Reifenberger \& von Deimling, 2015).

\section{Functional and Psychosocial Consequences of Brain Tumour}

Symptom onset for many people is sudden and has a clear neurological origin (e.g., seizures or speech disturbance), which usually leads to early contact with medical professionals and rapid diagnosis. However, when symptoms develop more gradually or are non-specific in nature (e.g., mood changes) medical investigations and diagnosis can be delayed or drawn out (Ownsworth et al., 2011). Treatment for brain tumour is lengthy and multifaceted, and may involve active monitoring, surgery, chemotherapy and radiotherapy; often used in combination. For well-defined and accessible tumours, treatment may be curative in nature through complete resection. Key treatment goals for people with high grade glioma are to increase time to progression (progression-free survival) and minimise adverse treatment effects to maximise quality of life (Lamborn et al., 2008). In this respect, there is a need to carefully balance the potential benefits of treatment for achieving disease control 
against the risks of treatment-related neurotoxicity and associated functional decline (Noggle \& Dean, 2013).

Functional impairments arising from brain tumour may relate to several mechanisms including compression and displacement effects, infiltration or invasion of surrounding brain tissue, disrupted flow of cerebrospinal fluid, raised intracranial pressure and treatment-related neurotoxicity (e.g., white matter abnormalities associated with chemotherapy). Mass effects refer to widespread damage or disruption to brain functions, which typically lead to more generalised functional impairment (e.g., global cognitive decline). Focal effects refer to more localised damage with functional deficits typically, although not exclusively, related to the tumour location; for example, left frontal tumours often affect speech production (MelladoCalvo \& Fleminger, 2009).

Although cognitive deficits are most marked during active treatment, these can persist long-term or emerge as late effects (e.g., delayed neurotoxic effects of radiotherapy). Cognitive impairments related to brain tumour arise from an interplay of physiological, medical, and psychological factors (see Noggle \& Dean, 2013). Accordingly, there is considerable variability in the neuropsychological profiles of people with brain tumour (Dwan, Ownsworth, Chambers, Walker \& Shum, 2015). Brain abnormalities evident from neuroimaging do not always manifest in cognitive effects; equally, people’s subjective reports of cognitive problems in daily life may not correspond with their neuroimaging or neuropsychological test results (Noggle \& Dean, 2013). Self-reported impairments have been found to be more closely related to health-related quality of life than cognitive test performance (Ownsworth, Hawkes, Chambers, Walker \& Shum, 2010).

In a study comparing eight cancer groups, people with brain tumour were found to report more neuro-cognitive symptoms (e.g., sensory and motor dysfunction, cognitive and communication deficits, headaches and seizures) than other cancers (Lidstone, Butters, Seed, 
Sinnot, Beynon, \& Richards, 2003). Brain tumour is also associated with emotional and behavioural changes, including apathy/initiation problems, verbal aggression and inappropriate behaviour (Simpson et al., 2015). In a large study $(n=363)$ investigating generalised anxiety and depression after brain tumour, Arnold et al. (2008) reported that $41 \%$ and $48 \%$ of people with brain tumour experienced clinical levels of depression and anxiety respectively. Females, people with lower education, and those with a premorbid psychiatric history were found to report significantly higher levels of anxiety and depression. Perhaps most strikingly, Arnold et al. found that individuals with low grade tumours (I-II) were more likely to develop generalised anxiety and depression than those with high grade tumours (IIIIV). Such findings suggest that individuals with a poorer prognosis for survival may not necessarily experience greater psychological distress than those with a good prognosis. Nonetheless, anxiety about dying, reported in up to $50 \%$ of people with brain tumour, is associated with poorer quality of life, fatigue, and depression (Pelletier, Verhoef, Khatri, \& Nagen, 2002).

The threat to life posed by brain tumour often propels people to consider their own mortality and the meaning and purpose of life. Existential well-being relates to people's sense of meaning, purpose, and satisfaction in life, and their relative levels of comfort or distress regarding death and suffering. (Ownsworth \& Nash, 2015). Participants in our qualitative research often expressed existential fears; for example, "Late at night is very hard, in the quiet of your room and it's dark. You go back into the past, you go into the future and you go over everything. It's like being on death row. (Ownsworth et al., 2011, p. 132). They were also concerned for family members, as follows: "Whether you're going to survive or not.... you want to be there for your children” (p. 128). We found that people with greater existential well-being were typically older age, higher on dispositional optimism, and had lower perceptions of threat and increased perceptions of controllability regarding their brain 
tumour. Further, optimism was related to existential well-being through a mediating effect of perceived controllability, suggesting that higher optimism may promote greater existential well-being by increasing people's focus on aspects of their illness that are controllable (Ownsworth \& Nash, 2015).

Family members have a primary role in supporting individuals to cope with the everyday impact of the illness and its treatment. Due to the trend for shorter stays in hospital and greater emphasis on outpatient care, family members are faced with a sudden life change with often very little time between their loved one’s diagnosis and discharge to adjust. Many feel underprepared and overwhelmed by their caregiving responsibilities and experience a high level of strain due to major changes to family roles and dynamics and uncertainty about the future (Ownsworth, Goadby \& Chambers, 2015). Not surprisingly, high rates of depression (31\%) and anxiety (59\%) have been reported in family caregivers (Pawl, Lee, Clark, \& Sherwood, 2013). We found that a higher level of caregiver strain was related to greater functional impairments in the person with brain tumour (Ownsworth, Henderson, Chambers \& Shum, 2009). Further, strain mediated the effects of the interpersonal and emotional difficulties on caregiver depression. Importantly, we also found that social support can buffer the impact of functional impairments; specifically, caregivers of people with severe functional impairments who were highly satisfied with their social support had better psychological wellbeing (Ownsworth, Chambers \& Henderson, 2010). Family members differ in their preferences for the type and timing of support received from professionals and their informal support networks. However, we found that most family caregivers valued access to information about brain tumour and its treatment from professionals as well as emotional support from professionals and their extended family and friends (Ownsworth, Goadby et al., 2015). 


\section{Psychological assessment considerations}

The functional, cognitive, behavioural and psychosocial difficulties outlined highlight the importance of multi-disciplinary assessment and management for people with brain tumour. In terms of psychology input, neuropsychological assessment can help to monitor people's cognitive status throughout the illness, with test results aiding the early detection of tumour recurrence (Meyers \& Brown, 2006). Supportive and timely feedback from such assessments can also provide valuable information to people with brain tumour and their family members. However, when deciding on the test battery both the sensitivity and potential burden of assessment needs to be considered due to fatigue and other distressing symptoms. Some brief cognitive screens lack sensitivity in detecting cognitive deficits after brain tumour (see Robinson, Biggs \& Walker, 2015). When a comprehensive neuropsychological assessment is not feasible or appropriate, a brief multi-faceted battery involving tests with demonstrated sensitivity is optimal (Dwan et al., 2015). In particular, the response assessment in neuro-oncology (RANO) working group and the International Cognition and Cancer Task Force (Wefel, Vardy, Ahles \& Schagen, 2011) recommend use of the Hopkins Verbal Learning Test-Revised, Trail Making Test, and Controlled Oral Word Association Test to monitor cancer-related changes in learning, memory, attention, processing speed and executive functioning. In our brain tumour research (see Ownsworth, Chambers et al., 2015) these three tests were included along with an estimate of pre-morbid IQ and measures of auditory attention (Digit Span) and visual memory (Rey Figure) in a 4045 minute test battery.

International quality standards also exist for cancer care facilities to routinely screen individuals and family members for distress and provide appropriate supportive care (Holland, Watson \& Dunn, 2011). A screening tool recommended for the brain tumour population is the National Comprehensive Cancer Network's Distress Thermometer, which 
involves a visual analogue scale and checklist of sources of stress and concerns. Although a score of $\geq 6$ has been identified as having optimal sensitivity and specificity for identifying distress in this population (Goebel \& Mehdorn, 2010), further clinical assessment is recommended for scores of $\geq 4$ (Trad et al., 2015). In terms of further assessment, the Depression Anxiety and Stress Scales and the Hospital Anxiety and Depression Scale have been validated for use in brain tumour (Ownsworth et al., 2008). For a more thorough assessment of mood, existential well-being and quality of life we have used the Montgomery Asberg Depression Rating Scale (structured clinical interview format), McGill Quality of Life Inventory and Functional Assessment of Cancer Therapy-Brain (Ownsworth, Chambers et al., 2015).

\section{Psychosocial interventions}

Psychosocial components of care focus on people’s understanding of their illness, their perceptions and reactions to the diagnosis and symptoms and the ways in which individuals and their family members cope with the illness. Supportive care interventions include education and information provision, multi-disciplinary rehabilitation, psychotherapy, support groups, family-based support, and palliative care or end-of-life support (Salander, 2010). Although there is a lack of literature on the role of neuro-oncology care coordinators and nursing practitioners, these professionals have an integral role in coordinating care and facilitating adjustment to diagnosis, treatment and end-of-life care issues. People with brain tumour and their family members often value the opportunity to discuss existential issues early in the illness rather than support only being offered at the palliative stage of care (Ownsworth et al., 2011). In an important development in personalised care, pilot research by Langbecker, Janda and Yates (2012) suggests that a brain tumour-specific question prompt list may help to reduce the unmet information and support needs. 
Until quite recently, most intervention research for the brain tumour population involved case-level descriptions of programs or services, such as nurse-led telephone support, support groups, and palliative care services (see reviews by Langbecker \& Janda, 2015; Salander, 2010). Over the past decade several controlled trials involving people with primary brain tumour have been conducted that provide preliminary support for the efficacy of videobased education (e.g., El-Jawahri et al., 2010), multi-disciplinary rehabilitation (Khan, Amatya, Drummond \& Galea, 2014), cognitive rehabilitation (Locke et al., 2008; Gehring et al., 2009; Zucchella et al., 2013) and psychotherapy (Ownsworth et al., 2014). For example, in a randomised controlled trial (RCT) involving 50 people with malignant glioma, ElJawahri et al. (2010) compared the effects of brief video-based education on medical care in advanced cancer combined with verbal narrative to verbal narrative only. Those receiving the video-based education demonstrated significantly greater knowledge of medical care. In a small RCT ( $n=19)$ involving people with brain tumour and their caregivers, Locke et al. (2008) evaluated the effects of cognitive rehabilitation (12 sessions of problem-solving therapy) relative to standard care. There were no significant differences on measures of quality of life, functional status, cognition, mood or fatigue. However, participants reported satisfaction with the program and there was good uptake of strategies learnt in the program.

More positive findings regarding the efficacy of cognitive rehabilitation were reported in two larger RCTs by Gehring et al. (2009) and Zucchella et al. (2013). Specifically, in a sample of 140 people with low grade glioma, Gehring et al. (2009) found significantly greater improvement on both subjective and objective indices of cognitive impairment following a brief cognitive rehabilitation intervention (6 $\mathrm{x} 2$ hour individual sessions) relative to a wait list condition. Similarly, Zucchella et al. (2013) found that individuals with brain tumour ( $n=$ 58) who received 16 sessions of individual cognitive rehabilitation after surgery showed greater cognitive gains than those receiving usual care after surgery. In a study involving 106 
patients with mixed glioma, Khan and colleagues (2014) found that people receiving individualised and comprehensive multi-disciplinary rehabilitation (2-3 times per week x 8 weeks) made greater overall functional gains than wait list controls. Although these RCTs broadly highlight the benefits of rehabilitation for improving cognitive and physical status, the objective functional gains observed were not accompanied by improvements in mood or quality of life at post-intervention or at the 3-6 months follow-up assessments.

In the first controlled trial of psychotherapy for people with brain tumour ( $n=50)$, we evaluated the effects of the Making Sense of Brain Tumour (MSoBT; Ownsworth, Chambers et al., 2015) program on mood and quality of life relative to a wait list condition. Conducted in people's own homes, the 10-session MSoBT program was guided by Sense of Coherence theory (Antonovsky, 1996) which proposes that when people are faced with adversity such as a serious illness, their well-being is enhanced when they understand what is happening to them (comprehensibility), believe they possess resources to manage the demands of the situation (manageability), and can find meaning in their life situation (meaningfulness). A combination of individual and family/couple sessions were delivered by a clinical psychologist to support people to understand the personal significance of their illness. The program was comprised of core (sessions 1, 2 and 10) and modularised components which supported the tailoring of therapy to people's goals and family situation (see Stewart \& Ownsworth, 2014). Common goals related to understanding and managing the cognitive, behavioural and emotional effects of brain tumour, exploring existential issues and the concerns of family members, and improving occupational and relationship functioning.

After controlling for covariates and baseline functioning, participants who received the MSoBT intervention reported significantly lower levels of depression on the Montgomery-Asberg Depression Rating Scale relative to wait list controls. The intervention group also reported significantly higher levels of existential well-being, functional well-being 
and global quality of life at post-assessment than the waitlist group. Further, at the 6-months follow-up assessment participants reported significantly lower levels of depression and stress, and higher existential well-being and quality of life relative to their pre-intervention functioning. Importantly, intervention outcomes were comparable for people with benign and malignant tumours. Having a family member involved in the program (60\% of all programs) was associated with lower depression and better social well-being at post-intervention (Ownsworth, Chambers et al., 2015).

Although these findings support the efficacy of in-home face-to-face psychotherapy for improving mental health and quality of life in individuals with brain tumour, this intervention format may not be feasible for broader translation into service delivery in the community due to therapist travel time and expenses. People with brain tumour face many barriers to accessing hospital or clinic-based counselling, such as inability to drive or use public transport due to poor physical health, cognitive impairment and financial strain. Those living in rural and remote areas face additional barriers to accessing brain tumour-specific therapy. As outlined in the next section, tele-health offers a potentially more flexible, accessible, and cost-effective option for providing psychological support to people with brain tumour and their family members.

\section{Pilot Intervention Research and Future Directions}

A key advantage of tele-health services is that psychological support can be provided without moving the support recipient or professional, thus overcoming barriers to participation in face-to-face programs (e.g., lack of transport, geographical isolation). In addition to voice dialogue, tele-health may entail educational material sent via email or normal post. There are several tele-health formats (e.g., telephone, Skype, video-conferencing, Internet-based resources); hence, there is a need to take into account people’s sensory-perceptual, language 
and cognitive functioning as well as their familiarity with technology, particularly for computer-mediated support. Pilot research into the feasibility of an interactive brain tumourspecific website by Piil, Joakobsen, Juhler and Jarden (2015) found that the website had relatively low levels of use by people with brain tumour. Interviews identified a major theme of “challenges and barriers” whereby people who were newly diagnosed often felt too overwhelmed by the change in their life situation to use the online resource. Some people experienced technological difficulties which in part were related to their cognitive impairments. Users expressed impatience with sites that were too difficult to use and delayed responses to questions from specialists. Nonetheless, caregivers valued the website as a resource and the opportunity to ask specialists questions. An RCT of Internet-based information provision and problem-solving support is currently underway in The Netherlands for people with glioma with mild to moderate depression and their caregivers. This trial which will help to further determine the feasibility and efficacy of Internet-based support (Boele et al., 2014).

Using a more conventional tele-health format, we trialled the feasibility and acceptability of telephone-based format of the MSoBT program (Jones, Ownsworth \& Shum, 2015). The 10-session program was delivered to four participants with brain tumour (1 benign; 2 low grade, 1 high grade). Participants were screened using a validated telephonebased cognitive screen to ensure adequate cognitive and communication skills and the therapist provided therapy materials via post or email prior to, and/or immediately after sessions to summarise session content. All four participants completed the 10 session program and endorsed high levels of therapeutic alliance. Participants perceived that having therapy sessions in their own home was a big advantage over travelling to a clinic due to physical symptoms and transportation issues. Outcome data for the program showed that overall mean depression and anxiety levels decreased from above clinical threshold to below 
threshold, and that quality of life improved at post- and follow-up relative to pre-intervention. An RCT of tele-based psychotherapy is now needed to investigate clinical efficacy and the factors influencing response to intervention.

There have also been promising developments in family-based support and training of health professionals. Whiting and colleagues (2012) developed and piloted a multi-tiered intervention approach for managing cognitive and behavioural difficulties related to brain tumour. This involved a combination of behavioural therapy and skills training intervention for a person with brain tumour, educating and training family members on how to manage challenging behaviour and a training workshop on behavioural management for health professionals. Each intervention showed positive effects in terms of decreasing target behaviours (evaluated in a single-case experimental design) and increasing knowledge of strategies. Although further research is needed to investigate clinical efficacy, these findings support the feasibility and utility of multi-level behavioural support interventions.

In conclusion, although there have been some key developments in psychosocial assessment and management, the development and evaluation of psychosocial interventions for people with brain tumour is still an emerging research area. There are currently insufficient controlled trials to provide Level I evidence concerning the efficacy of psychosocial interventions, as needed to develop clinical practice guidelines. Due to the integral role of family members in care, it is essential for interventions to address the information and emotional support needs of both the person with brain tumour and family members. 


\section{References}

Aldape, K., Zadeh, G., Mansouri, S., Reifenberger, G., \& von Deimling, A. (2015). Glioblastoma: Pathology, molecular mechanisms and markers. Acta Neuropathologica, 129, 829-48.

Antonovsky, A. (1996). The salutogenic model as a theory to guide health promotion. Health Promotion International, 11, 11-18.

Arnold, S. D., Forman, L. M., Brigidi, B. D., Carter, K. E., Schweitzer, H. A., Quinn, H. E., ... Raynor, R. H. (2008). Evaluation and characterization of generalized anxiety and depression in patients with primary brain tumors. Neuro-oncology, 10, 171-81. doi:10.1215/15228517-2007-057.

Australian Institute of Health \& Welfare (2014). Cancer in Australia: An overview. Cancer series no. 90. Cat. no. CAN 88. Canberra: AIHW.

Boele, F. W., Verdonck-de Leeuw, I. M., Cuijpers, P., Reijneveld, J. C., Heimans, J. J., \& Klein, M. (2014). Internet-based guided self-help for glioma patients with depressive symptoms: design of a randomized controlled trial. BMC Neurology, 14:81. doi:10.1186/1471-2377-14-81

El-Jawahri A, Podgurski LM, Eichler AF, Plotkin SR, Temel JS, Mitchell SL, ... Volandes, A. E. (2010). Use of video to facilitate end-of-life discussions with patients with cancer: a randomized controlled trial. Journal of Clinical Oncology, 28, 305-10. doi:10.1200/JCO. 2009.24.7502

Ferlay, J., Soerjomataram, I., \& Ervik, M. (2013). GLOBOCAN 2012 v1. 0, Cancer Incidence and Mortality Worldwide: IARC Cancer Base No. 10, International Agency for Research on Cancer.

Fuemmeler, B. F., Elkin, D. T., Mullins, L. L. (2002). Survivors of childhood brain tumors: Behavioral, emotional, and social adjustment. Clinical Psychology Review, 22, 547-585

Gehring, K., Sitskoorn, M.M., Gundy, C.M., Sikkes, S.A., Klein, M., Postma, T.J., Aaronson, N.K. (2009). Cognitive rehabilitation in patients with gliomas: A randomized, controlled trial. Journal of Clinical Oncology, 27, 3712-3722. doi:10.1200/JCO.2008.20.5765

Goebel, S., \& Mehdorn, H. M. (2010). Measurement of psychological distress in patients with intracranial tumours: The NCCN distress thermometer. Journal of Neuro-oncology, 104, 357-64. doi: 10.1007/s11060-010-0501-5.

Holland, J. C., Watson, M., \& Dunn, J. (2011). The IPOS new international standard of quality cancer care: integrating the psychosocial domain into routine care. Psychooncology, 20, 677-80. doi:10.1002/pon.1978

Jones, S., Ownsworth, T., \& Shum, D. (2015). Feasibility and utility of telephone-based psychological support for people with brain tumor: A single-case experimental study. Frontiers in Oncology, 5, 71, doi: 10.3389/fonc.2015.00071 
Lamborn, K. R., Yung, W. K., Chang, S. M., Wen, P. Y., Cloughesy, T. F., DeAngelis, L. M., ... Prados, M. D. (2008). Progression-free survival: An important end point in evaluating therapy for recurrent high-grade gliomas. Neuro-Oncology, 10, 162-170.

Langbecker, D., \& Janda, M. (2015). Systematic review of interventions to improve the provision of information for adults with primary brain tumours and their caregivers. Frontiers in Oncology, 5, 1-11. doi: 10.3389/fonc.2015.00001

Langbecker, D., \& Janda, M., \&Yates, P. (2012). Development and piloting of a brain tumour-specific question prompt list. European Journal of Cancer Care, 21, 51726.doi:10.1111/j.1365-2354.2012.01328.x

Lidstone, V., Butters, E., Seed, P.T., Sinnot, C., Beynon, T., \& Richards, M. (2003).

Symptoms and concerns amongst cancer outpatients: Identifying the need for specialist palliative care. Palliative Medicine, 17, 588-595.

Locke, D., Cerhan, J. H., Wu, W., Malec, J. F., Clark, M. M., Rummans, T. A., \& Brown, P. D. (2008). Cognitive rehabilitation and problem-solving to improve quality of life of patients with primary brain tumors: a pilot study. Journal of Supportive Oncology, 6, 383-91.

Louis, D. N., Ohgaki, H., Wiestler, O. D., Cavenee, W. K., Burger, P. C., Jouvet, A., \& Kleihues, P. (2007). The 2007 WHO classification of tumours of the central nervous system. Acta Neuropathologica, 114, 97-109.

Mellado-Calvo, N., \& Fleminger, S. (2009). Cerebral Tumours. In A. S. David, S. Fleminger, M. D. Kopelman, S. Lovestone \& J. D. Mellers (Eds.), Lishman's Organic Psychiatry: A Textbook of Neuropsychiatry (4th ed.). Singapore: Wiley-Blackwell.

Noggle, C. A., \& Dean, R. S. (Eds.) (2013). The neuropsychology of cancer and oncology. New York, NY: Springer Publishing Company.

Ostrom, Q.T., Gittleman, H., Liao, P., Rouse, C., Chen, Y., Dowling, J., .... Barnholtz-Sloan, J. (2014). CBTRUS Statistical report: Primary brain and central nervous system tumors diagnosed in the United States in 2007-2011. Neuro-oncology, 16(supp 4), iv1-iv63.

Ownsworth, T., Chambers, S., Hawkes, A., Walker, D., \& Shum, D. (2011). Making sense of brain tumour: a qualitative investigation of personal and social processes of adjustment. Neuropsychological Rehabilitation, 21, 117-37. doi:10.1080/09602011. 2010.537073

Ownsworth, T., Chambers, S., Stewart, E., Casey, L., Walker, D., \& Shum, D. (2014). Evaluation of the making sense of brain tumor program: a randomized controlled trial of a psychosocial intervention. Psycho-oncology, 24, 540-547. doi:10.1002/pon.3687.

Ownsworth, T., Hawkes, A., Chambers, S., Walker, D., \& Shum, D. (2010). Applying a biopsychosocial perspective to investigate factors related to emotional adjustment and quality of life for individuals with brain tumour. Brain Impairment, 11, 270-280. 
Ownsworth, T. L., Henderson, L., \& Chambers, S. (2010). Social support buffers the impact of functional impairments on caregiver psychological well-being in the context of brain tumor and other cancers. Psycho-Oncology, 19, 1116-1122.

Ownsworth, T.L., Henderson, L., Chambers, S., \& Shum, D. (2009). Functional impairments and caregiver depression in the context of brain tumour and other cancers: a mediating effect of strain. Brain Impairment, 10, 149-161.

Ownsworth, T., Little, T., Turner, B., Hawkes, A., \& Shum, D. (2008). Assessing emotional status following acquired brain injury: the clinical potential of the depression, anxiety and stress scales. Brain Injury, 22, 858-69. doi:10.1080/02699050802446697

Pawl, J., Lee, S. Y., Clark, P. C., \& Sherwood, P. R. (2013). Sleep characteristics of family caregivers of individuals with a primary malignant brain tumor. Oncology Nursing Forum, 40, 171-9.

Pelletier, G., Verhoef, M. J., Khatri, N., \& Nagen, N. (2002). Quality of life in brain tumor patients: the relative contributions of depression, fatigue, emotional distress and existential issues. Journal of Neuro-oncolgy, 57, 41-9. doi:10.1023/A:1015728825642

Piil, K., Joakobsen, J., Juhler, M., \& Jarden, M (2015). The feasibility of a brain tumour website. European Journal of Oncology Nursing, 19, 686-693.

Salander. P. (2010). Facilitating interventions and/or relationships in malignant brain tumours. Advanced Therapy, 27, 17-27.

Stewart, E., \& Ownsworth, T. (2014). Making Sense of Brain Tumour: A practical guide for therapists. Sydney: ASSBI Resources. ISBN: 978-0-9870477-7-9.

Van Meir, E. G., Hadjipanayis, C. G., Norden, A. D., Shu, H. K., Wen, P. Y., \& Olson, J. J. (2010). Exciting new advances in neuro-oncology: The avenue to a cure for malignant glioma. CA: A Cancer Journal for Clinicians, 60, 166-93.

Wefel, J. S., Vardy, J., Ahles, T., \& Schagen, S. B. (2011). International Cognition and Cancer Task Force Recommendations to harmonise studies of cognitive function in patients with cancer. Lancet Oncology, 12, 703-708. doi.org/10.1016/S14702045(10)70294-1

Whiting, D. L., Simpson, G. K., Koh E-S., Wright, K. M., Simpson, T., \& Firth, R. (2012). A multi- tiered intervention to address behavioural and cognitive changes after diagnosis of primary brain tumour: A feasibility study. Brain Injury, 26, 950-61.

doi:10.3109/02699052.2012.661912

Zucchella, C., Capone, A., Codella, V., De Nunzio, A. M., Vecchione, C., Sandrini, G., ... Bartolo, M. (2013). Cognitive rehabilitation for early post-surgery inpatients affected by primary brain tumor: a randomized, controlled trial. Journal of Neuro-oncology, 114, 93-100. doi:10.1007/s11060-013-1153-z 